\title{
O.S.P.
}

L'orientation scolaire et professionnelle

$48 / 4 \mid 2019$

Orientation et genre : approches psychosociales

\section{S'Orienter : un programme pour transformer les représentations sexospécifiques d'élèves sur les métiers et les professions}

S'Orienter: a program to transform gender stereotypes of professions

Patricia Dionne, Amélie Simard et Sylvain Bourdon

\section{OpenEdition}

Journals

Édition électronique

URL : https://journals.openedition.org/osp/11587

DOI : 10.4000/osp. 11587

ISSN : 2104-3795

Éditeur

Institut national d'étude du travail et d'orientation professionnelle (INETOP)

Édition imprimée

Date de publication : 1 décembre 2019

Pagination : DCXV-DCXXXV

ISSN : 0249-6739

Référence électronique

Patricia Dionne, Amélie Simard et Sylvain Bourdon, « S'Orienter : un programme pour transformer les représentations sexospécifiques d'élèves sur les métiers et les professions ", L'orientation scolaire et professionnelle [En ligne], 48/4 | 2019, mis en ligne le 01 décembre 2021, consulté le 18 mars 2022. URL : http://journals.openedition.org/osp/11587 ; DOI : https://doi.org/10.4000/osp.11587

Ce document a été généré automatiquement le 18 mars 2022.

(c) Tous droits réservés 


\title{
S'Orienter : un programme pour transformer les représentations sexospécifiques d'élèves sur les métiers et les professions
}

\author{
S'Orienter: a program to transform gender stereotypes of professions
}

\author{
Patricia Dionne, Amélie Simard et Sylvain Bourdon
}

\section{Introduction}

1 Plusieurs travaux sur les choix d'orientation ont mis en lumière le rôle des représentations qu'ont les jeunes et les adultes du marché du travail dans leurs choix tout au long de leur parcours (ex. Duru-Bellat, 2011). Ces représentations parfois limitantes du marché du travail sont construites dans un contexte historique et culturel où les inégalités de salaire et de possibilités professionnelles persistent dans plusieurs pays entre femmes et hommes (Bimrose, McMahon, \& Watson, 2019). C'est aussi le cas au Canada, où malgré de nombreuses politiques publiques visant à réduire ces inégalités, le salaire des professions où les femmes sont majoritaires, a tendance à être inférieur à celui des professions majoritairement exercées par des hommes, même avec une scolarisation comparable (Statistique Canada, 2017). Dans des emplois à niveaux égaux de scolarité, le salaire horaire des femmes correspond en moyenne à 87 $\%$ de celui touché par les hommes.

2 Certaines représentations sociales impliquant des conceptions stéréotypées liées au sexe peuvent affecter les choix d'orientation des personnes (Kergoat, 2014), parfois de manière non-consciente. Ces représentations sexospécifiques ${ }^{1}$ impliquent deux dimensions, l'une descriptive - qui concerne ce que les femmes et les hommes seraient et l'autre prescriptive, laquelle décrit ce qu'elles et ils devraient être, et implique une forme de sanction pour les personnes ne correspondant pas aux normes attendues (Heilman, 2012). Ces dimensions des représentations sexospécifiques peuvent limiter 
les aspirations professionnelles (Metz, Fouad, \& Ihle-Helledy, 2009) et, de facto les possibilités (Kergoat, 2014). Elles peuvent aussi entraîner des inégalités d'accès à certaines professions ; cela a été démontré en gestion, par exemple (Heilman, 2012). En outre, en milieu scolaire, malgré plusieurs interventions visant à modifier les représentations sexospécifiques sur certaines filières d'enseignement, la proportion d'élèves s'inscrivant dans des filières non traditionnelles demeure très faible (Vouillot, Blanchard, Marro, \& Steinbrukner, 2004). Dans ce contexte, notre équipe de recherche a développé au Québec le programme S'Orienter, qui vise à soutenir l'accompagnement d'un groupe d'élèves dans leur orientation vers des choix éducatifs et professionnels qu'elles et ils valorisent (Dionne, Simard, Bourdon, Supeno, \& Girardin, 2020). Ce programme de groupe vise également à favoriser chez les membres un rapport plus conscient aux représentations - notamment sexospécifiques - qui peuvent limiter leur capacité à choisir.

\section{Intervenir en groupe : transformer des représentations sexospécifiques}

3 Une recension des écrits scientifiques relatifs au domaine de l'orientation scolaire et professionnelle a permis de dégager que l'intervention de groupe aurait des retombées positives sur la transformation des représentations sexospécifiques des métiers et des professions. L'intervention de groupe serait en effet susceptible d'influencer les représentations en permettant la création d'un espace de parole et l'exposition des personnes participantes à une diversité de points de vue (Ali, Brown, \& Loh, 2017 ; Leclerc, 2019).

4 L'intervention en groupe aurait aussi une influence positive significative sur les dimensions associées au sentiment d'efficacité personnelle (SEP) tel que conçu par Bandura (1986), sur la prise de décision de carrière (Nota, Soresi, \& Ferrari, 2008 ; Scott \& Ciani, 2008), sur la conscience collective et sur l'affirmation de soi (Aguiar \& Conceição, 2015 ; Shea, Ma, Yeh, Lee, \& Pituc, 2009). Cela étant, si l'intervention en groupe semble propice au travail sur les représentations sexospécifiques, les méthodologies employées dans les recherches recensées sont principalement quasiexpérimentales ; elles permettent peu de comprendre en profondeur ce qui, dans l'activité d'un groupe, soutient le développement d'un rapport plus conscient des élèves aux représentations sexospécifiques de métiers ou de professions, ni ce qui en permet la transformation. Notre équipe a ainsi cherché à répondre à la question de recherche suivante : comment la participation à un programme de groupe, le programme S'Orienter, influence-t-elle d'une part le développement d'un rapport plus conscient des élèves à soi, aux autres et au monde, et d'autre part la transformation de représentations sexospécifiques de métiers ou de professions?

\section{S'Orienter : développer un rapport plus conscient à soi, aux autres et au monde et transformer des représentations}

5 L'analyse de l'influence du programme S'Orienter sur le développement d'un rapport plus conscient à soi, aux autres et au monde s'appuie sur une conception culturelle- 
historique de la conscience. Pour Vygotski (2013/1934), la conscience se développe dans un rapport matériel concret avec l'activité, médiatisée par des instruments développés culturellement et historiquement. Dans un programme de groupe visant l'orientation scolaire et professionnelle, ici S'Orienter, des instruments conceptuels liés à l'orientation (comme ceux d'intérêts, de valeurs, de compétences, de normes et de représentations sexospécifiques) peuvent être transmis par les personnes conseillères pour aider les personnes participantes à mettre à distance leurs propres façons de penser, d'agir ou de ressentir. Elles peuvent ensuite progressivement s'approprier ces instruments et en faire usage de manière consciente et volontaire dans leur rapport à soi, aux autres et à différents mondes, ici principalement les mondes éducatif et professionnel (Dionne, Saussez, \& Bourdon, 2017).

\section{Méthode}

\section{Personnes participantes et programme}

6 Notre équipe a élaboré le programme $S^{\prime}$ rriente $^{2}$ et l'a expérimenté dans quatre écoles secondaires et deux établissements postsecondaires auprès d'élèves de 15 à 26 ans $(n=76)$; six groupes d'intervention ont donc été organisés. Le tableau 1 résume le déroulement du programme, ses objectifs et ses principales stratégies d'animation.

Tableau 1 :Tableau synthèse du programme S'Orienter ${ }^{3}$ Table 1: Summary table of the S'Orienter program

\begin{tabular}{|c|c|c|c|c|}
\hline Session & Nom de l'activité & Thème(s) abordé(s) & Objectif & $\begin{array}{l}\text { Stratégies } \\
\text { d'animation } \\
\text { Leclerc, 2019) }\end{array}$ \\
\hline \multirow[t]{3}{*}{1} & Il était une fois moi & $\begin{array}{l}\text { Accueil des } \\
\text { membres } \\
\text { Notion d'intérêt }\end{array}$ & $\begin{array}{l}\text { Favoriser les } \\
\text { sentiments d'inclusion } \\
\text { et de confiance en } \\
\text { explorant un intérêt } \\
\text { professionnel }\end{array}$ & $\begin{array}{l}\text { Brise-glace et } \\
\text { prélude } \\
\text { Anecdote sur soi } \\
\text { Discussion } \\
\text { groupe }\end{array}$ \\
\hline & $\begin{array}{l}\text { Présentation } \mathrm{du} \\
\text { programme }\end{array}$ & $\begin{array}{l}\text { Modèle des } 5 \mathrm{C}^{4} \\
\text { Explication des } \\
\text { dimensions }\end{array}$ & $\begin{array}{l}\text { Présenter le } \\
\text { programme et les } \\
\text { tâches prévues afin de } \\
\text { créer une alliance de } \\
\text { travail optimale }\end{array}$ & $\begin{array}{l}\text { Exposé magistral } \\
\text { Discussion } \\
\text { groupe }\end{array}$ \\
\hline & $\begin{array}{l}\text { Mon choix, ma } \\
\text { difficulté : mon } \\
\text { objet, mon image }\end{array}$ & $\begin{array}{l}\text { Intérêt } \\
\text { professionnel, } \\
\text { choix d'objet et } \\
\text { liaisons entre les } \\
\text { objets et des } \\
\text { représentations } \\
\text { sexospécifiques }\end{array}$ & $\begin{array}{l}\text { Amorcer des } \\
\text { discussions collectives } \\
\text { en parlant de ses } \\
\text { intérêts, besoins et } \\
\text { représentations. }\end{array}$ & $\begin{array}{l}\text { Médiation (image } \\
\text { ou objet) } \\
\text { Discussion } \\
\text { groupe }\end{array}$ \\
\hline
\end{tabular}




\begin{tabular}{|c|c|c|c|c|}
\hline & $\begin{array}{l}\text { Entre ce que j'en } \\
\text { pense et ce qu'on } \\
\text { en dit }\end{array}$ & $\begin{array}{l}\text { Les représentations } \\
\text { du travail des } \\
\text { jeunes }\end{array}$ & $\begin{array}{l}\text { Discuter des } \\
\text { statistiques liées aux } \\
\text { représentations du } \\
\text { travail dans un climat } \\
\text { collaboratif favorisant } \\
\text { les auto-dévoilements }\end{array}$ & $\begin{array}{l}\text { Activité ludique } \\
\text { (jeu-questionnaire) } \\
\text { Débat } \\
\text { Petites équipes }\end{array}$ \\
\hline & Fermeture & $\begin{array}{lll}\text { Contenu } & \text { et } \\
\text { ambiance de la } \\
\text { rencontre }\end{array}$ & \begin{tabular}{|lll} 
Donner & & des \\
informations & & \\
nécessaires & à & la \\
progression & de & la \\
démarche. & & \\
dél
\end{tabular} & $\begin{array}{lll}\begin{array}{l}\text { Tour de table } \\
\text { question à }\end{array} & \text { la } \\
\text { cantonade } & & \\
& & \\
\text { Discussion } & & \text { en } \\
\text { groupe } & & \end{array}$ \\
\hline \multirow{3}{*}{2} & À la source & $\begin{array}{l}\text { L'information } \\
\text { scolaire } \\
\text { professionnelle }\end{array}$ & $\begin{array}{l}\text { Clarifier les ressources } \\
\text { personnelles et } \\
\text { contextuelles liées à la } \\
\text { recherche } \\
\text { d'information afin de } \\
\text { générer des } \\
\text { possibilités }\end{array}$ & $\begin{array}{l}\text { Synthèse d'idées } \\
\text { Discussion en } \\
\text { groupe } \\
\text { Activité écrite } \\
\text { (schématisation) }\end{array}$ \\
\hline & $\begin{array}{l}\text { Un besoin, } \\
\text { plusieurs } \\
\text { possibilités }\end{array}$ & $\begin{array}{l}\text { Lien entre besoins } \\
\text { et rhoix } \\
\text { professionnels }\end{array}$ & $\begin{array}{lr}\text { Identifier ses besoins } \\
\text { dans le choix des } \\
\text { intérêts } & \\
\text { professionnels } & \text { à } \\
\text { travers } & \text { les } \\
\text { rétroactions } & \text { entre } \\
\text { pairs } & \end{array}$ & $\begin{array}{l}\text { Activité écrite } \\
\text { (schématisation) } \\
\text { Retour en groupe }\end{array}$ \\
\hline & Fermeture & $\begin{array}{l}\text { Les moments forts } \\
\text { La prochaine } \\
\text { rencontre }\end{array}$ & $\begin{array}{l}\text { Créer un espace de } \\
\text { discussion sur son } \\
\text { appréciation afin } \\
\text { d'ajuster la suite de la } \\
\text { démarche }\end{array}$ & 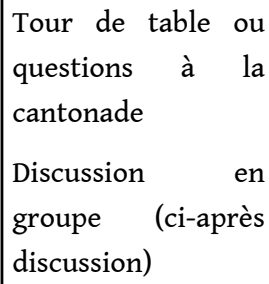 \\
\hline \multirow[b]{2}{*}{3} & $\begin{array}{l}\text { Mes choix ont de la } \\
\text { valeur }\end{array}$ & \begin{tabular}{|lr|}
\multicolumn{2}{l}{ Concept de valeur } \\
Liens avec les \\
concepts & de \\
besoins & et \\
d'intérêts & en \\
contexte de & choix \\
éducatifs & et \\
professionnels &
\end{tabular} & $\begin{array}{l}\text { Apprendre à parler de } \\
\text { soi et à respecter la } \\
\text { parole des autres à } \\
\text { travers l'identification } \\
\text { des valeurs et les } \\
\text { influences effectives } \\
\text { de celles-ci sur les } \\
\text { choix professionnels }\end{array}$ & $\begin{array}{l}\text { Activité ludique } \\
\text { (jeu de cartes) } \\
\text { Petites équipes }\end{array}$ \\
\hline & $\begin{array}{l}\text { L'histoire de mes } \\
\text { victoires }\end{array}$ & $\begin{array}{l}\text { Concept } \\
\text { d'aptitudes }\end{array}$ & $\begin{array}{l}\text { Identifier des } \\
\text { ressources internes } \\
\text { (dont les aptitudes) et } \\
\text { externes avec le } \\
\text { soutien des membres }\end{array}$ & $\begin{array}{l}\text { Activité écrite } \\
\text { (tableau synthèse) }\end{array}$ \\
\hline
\end{tabular}




\begin{tabular}{|c|c|c|c|c|}
\hline & Retour/Fermeture & & $\begin{array}{l}\text { Boucler la rencontre, } \\
\text { donner les consignes } \\
\text { pour la prochaine } \\
\text { session }\end{array}$ & $\begin{array}{lll}\text { Tour de table ou } \\
\text { questions à } \\
\text { cantonade } \\
& \\
\text { Discussion } & & \end{array}$ \\
\hline \multirow{4}{*}{4} & $\begin{array}{l}\text { Moi, } \\
\text { professionnel.le ?! }\end{array}$ & $\begin{array}{l}\text { Les représentations } \\
\text { pouvant limiter et } \\
\text { influençant nos } \\
\text { choix pour la } \\
\text { présentation de soi }\end{array}$ & $\begin{array}{l}\text { Se décrire avec une } \\
\text { carte professionnelle } \\
\text { en approfondissant sa } \\
\text { compréhension des } \\
\text { représentations } \\
\text { limitantes }\end{array}$ & $\begin{array}{l}\text { Médiation ou } \\
\text { création manuelle } \\
\text { (cartes } \\
\text { professionnelles) } \\
\text { Discussion }\end{array}$ \\
\hline & 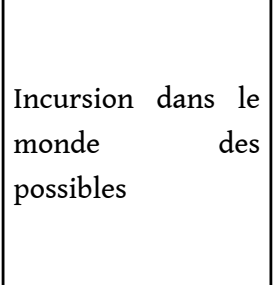 & $\begin{array}{l}\text { La recherche } \\
\text { d'information } \\
\text { scolaire et } \\
\text { professionnelle }\end{array}$ & $\begin{array}{l}\text { Réaliser une } \\
\text { recherche } \\
\text { informatisée } \\
\text { d'information scolaire } \\
\text { et professionnelle } \\
\text { (ISEP) }\end{array}$ & $\begin{array}{l}\text { Recherche } \\
\text { individuelle sur } \\
\text { ordinateur avec } \\
\text { activité écrite } \\
\text { de sistes à consulter } \\
\text { Discussion }\end{array}$ \\
\hline & $\begin{array}{l}\text { Tout le monde } \\
\text { m'en parle } \\
\text { (activité à la carte) }\end{array}$ & $\begin{array}{l}\text { Les représentations } \\
\text { présentes dans le } \\
\text { paysage sémiotique } \\
\text { les environnant }\end{array}$ & $\begin{array}{l}\text { Mener une recherche } \\
\text { en équipe pour } \\
\text { stimuler les prises de } \\
\text { conscience sur les } \\
\text { représentations dans } \\
\text { leur environnement } \\
\text { quotidien }\end{array}$ & $\begin{array}{l}\text { Activité ludique } \\
\text { (rallye) } \\
\text { Petites équipes }\end{array}$ \\
\hline & Fermeture & Bilan & $\begin{array}{l}\text { Donner les consignes } \\
\text { liées à la dernière } \\
\text { rencontre et poser les } \\
\text { bases de la fin du } \\
\text { processus. }\end{array}$ & $\begin{array}{lll}\text { Tour de table ou } \\
\text { questions à la } \\
\text { cantonade } & & \\
\text { Discussion } & & \end{array}$ \\
\hline \multirow{3}{*}{5} & $\begin{array}{l}\text { Moi avant, toi } \\
\text { maintenant }\end{array}$ & $\begin{array}{l}\text { Le bilan des } \\
\text { apprentissages } \\
\text { réalisés pendant la } \\
\text { démarche }\end{array}$ & $\begin{array}{l}\text { Soutenir les prises de } \\
\text { conscience au regard } \\
\text { des activités réalisées } \\
\text { dans la démarche }\end{array}$ & $\begin{array}{l}\text { Création manuelle } \\
\text { (collage) } \\
\text { Discussion }\end{array}$ \\
\hline & $\begin{array}{lrr}\text { Repères } & \text { dans } & \text { le } \\
\text { monde } & \text { des } \\
\text { possibles } & & \end{array}$ & \begin{tabular}{ll}
\multicolumn{2}{l}{ Le plan d'action } \\
La & recherche \\
d'ISEP &
\end{tabular} & \begin{tabular}{|lrl} 
Produire un & plan \\
d'action & & pour \\
concrétiser & leurs \\
choix en ciblant & des \\
sources externes.
\end{tabular} & $\begin{array}{l}\text { Activité écrite } \\
\text { (tableau synthèse) } \\
\text { Recherche sur } \\
\text { internet (au besoin) }\end{array}$ \\
\hline & $\begin{array}{l}\text { Merci à moi, merci } \\
\text { à toi... }\end{array}$ & $\begin{array}{l}\text { La reconnaissance } \\
\text { de sa contribution }\end{array}$ & $\begin{array}{l}\text { Clore le processus en } \\
\text { remerciant chaque } \\
\text { membre de manière } \\
\text { personnalisée }\end{array}$ & $\begin{array}{l}\text { Tour de table } \\
\text { Grand groupe }\end{array}$ \\
\hline
\end{tabular}




\begin{tabular}{|c|c|c|c|c|}
\hline Optionnel & Sur le web & $\begin{array}{l}\text { Tous contenus } \\
\text { apportés par les } \\
\text { membres faisant } \\
\text { suite aux sessions }\end{array}$ & $\begin{array}{l}\text { Soutenir les membres } \\
\text { entre les sessions }\end{array}$ & $\begin{array}{l}\text { Création d'un } \\
\text { forum ou d'une } \\
\text { page internet }\end{array}$ \\
\hline $\begin{array}{l}\text { Activité en } \\
\text { surplus ou } \\
\text { à la carte }\end{array}$ & $\begin{array}{l}\text { Moi entre deux } \\
\text { mondes... }\end{array}$ & $\begin{array}{l}\text { La formation d'un } \\
\text { rêve professionnel }\end{array}$ & $\begin{array}{l}\text { Comprendre le } \\
\text { processus de } \\
\text { formation d'un rêve/ } \\
\text { intérêt professionnel } \\
\text { en étant soutenu par } \\
\text { les autres membres du } \\
\text { groupe }\end{array}$ & \begin{tabular}{|lr} 
Imagerie & mentale \\
guidée & par la \\
lecture & d'une \\
histoire & \\
& \\
Discussion &
\end{tabular} \\
\hline
\end{tabular}

7 Pour le présent article, nous avons fait le choix de présenter les résultats relatifs à deux des groupes du secondaire, impliquant au total 22 élèves, car ils décrivent les retombées particulières du programme sur des jeunes qui font une primo-orientation. Les caractéristiques de ces sites et des personnes participantes sont présentées au tableau 2.

Tableau 2 : Caractéristiques des sites et des membres Table 2: Sites and Members Characteristics

\begin{tabular}{|c|c|c|}
\hline Caractéristiques & Site 1 & Site 2 \\
\hline $\begin{array}{l}\text { École } \\
\text { Recrutement }\end{array}$ & $\begin{array}{l}\text { École située en milieu } \\
\text { rural } \\
\text { Participation requise : } \\
\text { programme réalisé sur } \\
\text { les heures de classe. }\end{array}$ & $\begin{array}{l}\text { École située en milieu urbain (moyenne ville) } \\
\text { Participation volontaire : offert à des membres } \\
\text { d'un programme de prévention du décrochage } \\
\text { scolaire réalisé en collaboration avec une } \\
\text { ressource communautaire }\end{array}$ \\
\hline Niveau de scolarité & $\begin{array}{l}\text { Formation aux métiers } \\
\text { semi-spécialisés (FMS) }\end{array}$ & $3^{\mathrm{e}}$ et $4^{\mathrm{e}}$ secondaire \\
\hline Genre déclaré & $\begin{array}{l}6 \text { hommes } \\
9 \text { femmes }\end{array}$ & 5 femmes \\
\hline $\begin{array}{l}\text { Appartenance } \\
\text { culturelle } \\
\text { éthique }\end{array}$ & $\begin{array}{l}\text { Personnes caucasiennes } \\
\text { nées au Québec }\end{array}$ & $\begin{array}{l}3 \text { femmes nées en Afghanistan } \\
1 \text { femme née au Québec de parents afghans } \\
1 \text { femme née au Québec de parents africains }\end{array}$ \\
\hline
\end{tabular}

8 Le programme S'Orienter s'étale sur cinq rencontres de deux heures et s'appuie sur un modèle de développement du pouvoir d'agir en counseling de carrière (Chronister, McWhirter, \& Forrest, 2005) fondé sur cinq composantes : 1) la collaboration ; 2) la compétence ; 3) la considération du contexte influençant les aspirations professionnelles ; 4) la conscience critique et 5) la communauté. Ce modèle, qui reconnaît les rapports de pouvoir asymétriques entre les hommes et les femmes, a d'abord été opérationnalisé lors d'interventions auprès de femmes vivant une situation de violence conjugale. Pendant la mise en œuvre du programme, plusieurs instruments 
conceptuels du domaine de l'orientation éducative et professionnelle étaient introduits ; parmi ceux transmis par la conseillère d'orientation (CO), plusieurs concernaient la connaissance de soi (ex. intérêts, valeurs, compétences, aspiration) et la connaissance de l'information scolaire et professionnelle. Des ateliers visaient plus spécifiquement la transmission d'informations sur les représentations sexospécifiques du marché du travail et la mise en débat de celles-ci ; les éléments de connaissance de soi et du marché étaient mis en lien avec ces représentations.

\section{Collecte de données}

Des données qualitatives de diverses natures ont été collectées. Toutes les rencontres de groupe, animées par une $\mathrm{CO}$, ont d'abord été filmées. Une observatrice présente à toutes les rencontres a également pris des notes sur le déroulement de ces dernières. Une semaine après la fin du programme, un groupe de discussion a été organisé et filmé pour aborder principalement les thèmes suivants : les moments forts du programme et du groupe, les représentations de monde de travail, les choix d'orientation et les apprentissages que les membres considéraient avoir réalisés à la suite de leur participation au programme, plus spécialement les apprentissages pouvant être liés aux dimensions du modèle de Chronister et al. (2005). Des entrevues semi-dirigées individuelles ont enfin été réalisées trois mois après la fin du programme autour des mêmes thèmes que ceux abordés en groupe, mais un accent était mis sur les actions d'orientation faites depuis la fin du programme.

\section{Traitement des données}

10 Les verbatim des propos tenus par les personnes participantes ${ }^{5}$ lors des rencontres de groupe pendant le programme, lors du groupe de discussion post-programme sur chacun des sites et lors des entretiens individuels (site $1=15$; site $2=5$ ) ont été intégrés dans le logiciel NVivo 12. À la suite d'une analyse thématique à deux échelles, celle du groupe et celle des personnes participantes, une première matrice en condensés a été produite pour générer un découpage par rencontre et cibler, au cours du processus, les instruments conceptuels (ex. valeurs, conscience critique, représentations) transmis dans chaque groupe par la CO. Cela permettait par exemple de voir l'écart entre le programme tel que conçu par écrit et celui qui avait été réalisé avec les groupes. Une attention particulière a été portée aux moments de discussion des représentations sexospécifiques des métiers et des professions.

11 Dans une deuxième matrice, nous avons codé pour chacune des personnes participant au programme S'Orienter les manifestations discursives permettant de dégager un rapport plus conscient 1) à ces représentations et éventuellement à la transformation de celles-ci et 2) à l'influence potentielle de ces représentations sur les choix professionnels. Les résultats sont présentés ci-après selon le site d'intervention. Ils illustrent d'abord, grâce à des extraits de verbatim de l'intervention en groupe, comment les concepts ont été transmis et les représentations, mises en débat dans le cadre du programme. Puis, des extraits des groupes de discussion et entrevues individuelles sont présentés pour situer à l'échelle des personnes participantes l'influence de la participation au programme. 


\section{Résultats Site 1}

\section{Mise en débat des représentations sexospécifiques}

Dans le programme S'Orienter, le concept de représentations sexospécifiques est introduit directement à la première rencontre lors de la présentation du programme et le thème est repris explicitement à chacune des rencontres subséquentes. Sur le site 1 , lors de la première rencontre, la majorité des membres soutient que les hommes et femmes peuvent choisir et exercer n'importe quel métier ou profession. Si des représentations sexospécifiques affectent leur choix professionnel, ce processus est non-conscient au début du programme. Des nuances sont néanmoins graduellement introduites par les membres dès la rencontre 2, où une vision plus stéréotypée associée à ce que les hommes seraient - est amenée par Frank, qui affirme que ce sont les hommes qui seraient les mieux placés pour faire des métiers de la construction ou des métiers du domaine agricole. À la suite de cette déclaration, un autre participant, Nathan, l'invite à se taire, ce qui laisse penser que ce dernier perçoit davantage un risque à émettre une opinion "non désirable " socialement dans le contexte d'un programme où est valorisée l'équité des possibilités professionnelles :

Frank : Je suis sûr que c'est les gars. Je suis sûr que c'est eux qui sont plus constructifs [associé aux métiers de la construction].

Nathan : Bien non, non, Frank, chut, chut.

Immédiatement, la CO invite les autres membres, et particulièrement les jeunes femmes, à verbaliser leurs pensées et émotions sur le sujet. Même si certaines personnes sont hésitantes, le débat leur permet de discuter de représentations qui peuvent être véhiculées dans leur contexte :

$\mathrm{CO}$, reprenant les mots de Frank : « [Les gars] qui sont plus associés aux domaines de la construction. » Léonie et Jasmine, qu'est-ce que vous en pensez, de ça?

Léonie : Moi, je ne sais pas là.

$\mathrm{CO}$ : Êtes-vous d'accord? Est-ce que ça vous choque?

Jasmine : Je ne suis pas d'accord.

Frank : Veux-tu savoir ? Ce n'est pas une fille qui va aller dans un champ pour aller récolter, exemple, du blé.

Adeline : Je l'ai déjà fait!

Charlie : Il y en a vraiment [qui le font].

Phillipe : Bien, oui ! Elle n'a rien qu'à aller conduire le tracteur, puis foncer dans le champ. Frank : Non, mais...

Dans le même esprit, à la rencontre 4 lors de l'activité Tout le monde m'en parle, la CO demande aux membres de faire le tour de l'école pour photographier tout ce qui est visible sur les murs (ex. affiches, peintures) et pour en dégager ensuite les liens avec les représentations sexospécifiques. La CO propose d'analyser les publicités et les mots en termes de "stéréotypes" ou "d'étiquettes " associées au fait d'être une fille ou un garçon, invite les membres du groupe «à regarder c'est quoi le message derrière ? D'où ça vient ? ». L'activité suscite un débat mettant en lumière les inégalités dans les publicités disponibles : une affiche de contraception masculine est notamment trouvée seulement dans les toilettes des filles. Le groupe échange sur les inégalités que cela sous-tend quant à la responsabilité de la contraception, et plus largement celle de la planification familiale. La CO élargit la discussion aux représentations sociales des rôles selon le sexe :

$\mathrm{CO}$ : Ils mettent ça dans la toilette des filles, là. [...] 
Léonie : c'est comme si ça serait nous qui faudrait s'occuper des trucs... [rires].

Jasmine : Oui, mais ce n'est pas juste à nous, là.

Adeline : Effectivement.

Léonie : Ils devraient en mettre dans les [toilettes des] gars.

Jasmine : C'est ça.

Léonie : On va décrocher l'affiche, puis on va aller dans la toilette des gars. [...]

$\mathrm{CO}$ : Mais ce n'est pas la première fois que je vois ça dans des toilettes comme ça. On est dans une société qui responsabilise beaucoup les filles et qui déresponsabilise un petit peu les garçons. Frank : Oui. (Rencontre 3)

\section{Retombées}

L'analyse des manifestations discursives permet d'observer qu'en plus d'avoir permis à la majorité des élèves de clarifier leurs choix d'orientation, les discussions de groupe semblent avoir suscité une mise à distance des représentations sexospécifiques liées à des métiers ou professions. Les membres du groupe sur le site 1 sont plus critiques devant l'argument du manque de capacités, qui tend à éloigner les femmes des métiers jugés "plus difficiles physiquement». Alors qu'au début du programme, les membres indiquaient qu'il n'existait pas de différences dans les possibilités professionnelles pour les hommes ou les femmes, ils et elles semblent avoir davantage conscience, après le programme, de l'influence potentielle des représentations véhiculées dans leur contexte social, comme le laisse voir cet extrait du groupe de discussion final :

CO : Donc, il y a des métiers juste de gars, des métiers juste de filles? Frank : Non, ce n'est pas vrai ça. Tous les métiers, tous les gars puis toutes les filles peuvent [les faire]. Adeline : S'ils veulent.

CO : Donc ce préjugé-là, de métiers de filles ou de métiers de gars, vous n'êtes pas d'accord.

Marie-Jeanne : C'est comme chez nous... Je vais te donner comme exemple, moi, je vivais sur une ferme, tout le monde me disait : " ah, ça, c'est gars. » Puis ils m'embarquaient là-dedans. "Une fille ne sera pas capable de faire la job de fermier, de traire les vaches, de conduire les tracteurs, de ramasser des grosses roches. » [...] Mais au contraire là, une fille peut bien faire la job que le gars fait.

$\mathrm{CO}$ : Les filles sont capables. Est-ce qu'il y en a d'autres milieux, comme la ferme, qui pourraient être typiquement gars?

Marie-Jeanne : Comme à la job de Frank et d'Antoine, dans le bois. Il y a des filles qui travaillent là-dedans, puis il y a des gars qui disent : " ah non, tu ne seras jamais capable de faire ça, tu vas t'épuiser bien trop vite. " Puis ma tante travaille làdedans.

Élise : Il y a des gars qui travaillent en coiffure.

Frank : Il n'y a pas de : «Ah, tu ne seras pas capable » Non, si tu aimes ça, fais-le. Puis tu n'aimes pas ça, bien fais-le pas. (Groupe de discussion)

16 À la suite du programme S'Orienter, certaines personnes clarifient ou envisagent des choix qu'elles n'osaient pas avant les rencontres du groupe. Jasmine, qui vivait des relations personnelles abusives avant sa participation au programme, choisit d'en parler pour la première fois dans le groupe ; elle nomme qu'elle ose se prononcer sur des enjeux importants pour elle. Dans son entrevue individuelle, trois mois après sa participation, elle dit :

Je me sens mieux à dire ce que j'ai à dire. Je suis moins enfermée sur moi-même, je dis plus mon opinion. Je prends beaucoup plus la parole. [J'ai appris à] pas me laisser faire, puis à montrer ce que je suis. [...] Là, je suis plus confiante. [...] Puis ça m'a plus dirigée vraiment, vers ce que j'aime faire. (Jasmine) 
17 L'adolescente ajoute qu'elle veut se lancer en affaires et gérer sa propre entreprise, ce qui semblait difficile à envisager pour elle avant sa participation. En lien avec les discussions sur les représentations sexospécifiques, ce travail combinerait un travail parfois difficile physiquement auprès des animaux et la gestion d'une petite entreprise. Le centre de toilettage, « ça serait mon centre, qui serait plus à moi, mes personnes... mes responsabilités ».

\section{Résultats Site 2}

\section{Mise en débat des représentations sexospécifiques}

Sur le site 2, l'implantation du programme S'Orienter est marquée par une difficulté, plus particulièrement chez les jeunes femmes, à affirmer ${ }^{6}$ leurs intérêts. Dès la première rencontre, elles hésitent à choisir où et comment s'asseoir dans le local. Pour réaliser l'activité Mon choix, ma difficulté : mon objet, mon image, elles doivent choisir des images pour décrire un intérêt lié à leur orientation, et trouvent difficile de le faire. La CO insiste sur le concept de droit à plusieurs reprises dans la rencontre : « vous avez le droit de vous parler entre vous ", "vous avez le droit de vous poser des questions si vous avez des doutes ", " vous pouvez ne pas être d'accord avec moi ». Elle affirme également de manière explicite la valeur de la parole des membres : «Moi, je vous fais confiance, je pense que vous le savez ».

La CO donne aux membres du groupe le droit à une parole critique ; ce droit est réitéré à toutes les rencontres. De même, dans l'activité Présentation du programme à la rencontre 1, la CO explique le concept de conscience critique et précise que les rencontres permettront de prendre le temps de « remettre en question » la manière de faire des choix d'orientation : «Est-ce que j'ai vraiment envie de faire ça ? D'où ça vient ?" Des liens explicites sont faits avec l'environnement comme source potentielle d'influence des choix. La CO donne l'exemple de l'influence des parents; des échanges s'engagent sur les aspirations scolaires que des parents ayant immigré au Québec peuvent avoir pour leurs enfants et sur la pression que cela suscite :

Parisa : Ses parents, mes parents, c'est parce qu'eux n'avaient pas vraiment accès à l'éducation [...] ils auraient aimé devenir des personnes admirables, avoir des jobs prestigieuses. Nous, on a la chance d'avoir de l'éducation gratuitement, ils veulent que t'aime ça, si je peux dire. C'est comme s'ils voulaient que tu fasses ça pour eux autres un peu. [...] Mes parents disent souvent « parce que si tu deviens docteure, tu vas devenir quelqu'un d'admirable, et tu auras une bonne condition de vie ».

$\mathrm{CO}$ : alors, est-ce qu'on a le droit de faire d'autre chose que ce que nos parents ont prévu pour nous ? Est-ce qu'on a le droit ? [...] Zahra : C'est aussi un peu à cause de la culture, des traditions. On est quatre Afghanes, puis comme Parisa dit, [on a] la pression des parents qui n'ont pas eu la chance d'avoir l'éducation, et que nous on l'a [la chance].

Les jeunes femmes discutent alors du fait que certaines professions plus prestigieuses, particulièrement médecin pour les femmes et ingénieur pour les hommes, sont plus valorisées dans leur milieu familial. Les professions souhaitées pour les femmes sont clairement identifiées dans leur environnement. Les membres dévoilent progressivement une double contrainte dans leur choix professionnel : faire ce qu'elles aiment, mais devoir se conformer aux aspirations de leur famille. Certaines ajoutent que cette contrainte de réussite dans les études est aussi assortie d'une pression de se 
marier et d'être une bonne mère : «Je sais que c'est mon choix, mon projet, mais on dirait qu'il y a quelque chose qui me tire [retient] par ma famille ", dit Zahra. À la rencontre 5, en faisant l'activité Plan d'action, elle réalise qu'en plus de devoir réussir dans ses études et professionnellement, elle sent la pression de fonder une famille : « le fait de se marier puis avoir des enfants, c'est eux [mes parents] aussi ».

21 Après la rencontre 2, une rupture temporaire survient dans l'implantation du programme S'Orienter : les membres s'absentent. La CO se présente trois fois en vain sur les lieux. Puis, alors que l'équipe de recherche considère mettre fin à l'intervention sur ce site, quatre jeunes filles se mobilisent pour dire à la direction de l'organisme qui accueille le programme de prévention au décrochage qu'elles ont peur que le groupe s'arrête, et qu'elles veulent poursuivre les rencontres, importantes pour leur orientation professionnelle. Elles semblent progressivement s'approprier le droit de prendre la parole à propos d'un service qui leur est offert. Parisa souligne que les ateliers l'ont aidée à réaliser pourquoi elle ne s'affirmait pas en lien avec le choix scolaire auquel elle aspirait. Elle précise l'aspect insécurisant pour elle de l'approche mobilisée par la CO. En cohérence avec le modèle de Chronister et al. (2005), la CO favorise la collaboration et la conscience critique. Elle demande aux membres de prendre la parole et soutient l'importance de faire confiance à la valeur de leurs propres émotions et pensées, tout en suscitant la discussion de celles-ci pour ouvrir à de nouveaux possibles. Cette façon de faire contraste avec les rapports sociaux dont les membres ont l'habitude. La discussion amène aussi chez Pauline l'amorce d'une critique sur l'imposition des choix, qu'elle sent dans certains des milieux sociaux qu'elle fréquente :

$\mathrm{CO}$ : Non seulement, tu savais ce que tu voulais, maintenant tu sais ce que tu veux et il y a quelque chose qui s'est solidifié. Je pense que maintenant, tu sais que tu as le droit de vouloir ça.

Parisa : Oui, oui, oui, c'est vrai.

Zahra : [en parlant à Parisa] Oui, tu as droit à l'erreur.

Parisa : Comme souvent, j'entends des personnes qui veulent m'aider à faire un choix en me demandant «Qu'est-ce que tu aimes ? » et en me disant « fais ce que tu aimes ». [...] J'aime ça, ça, ça, mais ça ne va pas m'aider plus si quelqu'un me demande ce que j'aime. » Toi, tu vas m'amener à faire : «Ah ! c'est ça que j'aime. » J'ai aimé ta manière d'y arriver, c'était plus aidant. Je réalisais que les activités, elles étaient toutes par rapport à moi, elle m'aidait à voir pourquoi je ne m'affirmais pas à faire ce choix-là. Merci.

Pauline : Le fait que tu nous aies laissées libres, aussi, de ce qu'on pouvait faire puis de comment on pouvait voir les choses aussi. Vu que souvent, les endroits où l'on va, ils ne nous laissent pas vraiment le choix de voir comment on pense, qu'il y a différents choix, qu'il y a différentes manières de voir.

Parisa : La première fois, c'était différent. Des fois, j'étais : « Ah ! Mais pourquoi elle nous dit ça ? " Elle ne me dit pas : " Ça veut dire ça, il faut que tu écrives ça. » Des fois, parce que j'étais perdue, puis je voulais...

$\mathrm{CO}:$ C'est insécurisant?

Parisa : Oui. Je voulais quelqu'un qui me guiderait dans comment répondre, puis vous étiez : «C'est comme tu le perçois. » J'étais : «Ah! » (Rencontre 5)

\section{Retombées}

Au cours de l'activité du programme S'Orienter, à l'instar de ce qui a été constaté sur le site 1 , les membres du site 2 arrivent progressivement à prendre conscience de représentations - ou, plus largement, des normes - qui influent sur leur parcours 
scolaire ou professionnel. Elles développent un rapport conscient et critique à celles-ci. Dans l'extrait du groupe de discussion final, l'une des membres confie qu'elle est exposée à des messages parfois contradictoires et sexospécifiques sur ses possibilités d'orientation :

Les médias, parfois ils mettent des bons messages : « ah OK, tu peux être tout ce que tu veux, il n'y a pas de métiers de femmes ou d'hommes. » Puis des fois, il y a sur les photos, juste admettons des hommes qui sont ingénieurs.

De même, les manifestations discursives des participantes, dans leurs entrevues individuelles, laissent envisager qu'elles se donnent davantage le droit comme femmes de réaliser un choix professionnel qui les intéresse, tout en étant conscientes de la pression familiale qui pèse sur elles à différents égards, dont la réussite de leurs études :

Zahra : Ce que j'ai retenu, c'est le fait que peu importe ce que tu veux faire, il n'y a pas de limite. Ça ne veut pas dire que parce que tu es un garçon ou une fille que tu ne peux pas le faire. Il n'y a rien qui t'empêche [de] faire ce que tu veux.

Pauline : Ce que j'ai retenu, c'était que c'est notre vision des choses à nous qui compte vraiment, il ne faut pas se fier aux autres ou admettons à une bonne réponse. La manière que tu vois les choses, toi-même, ça peut être bon aussi. Tu n'es pas obligée de toujours te remettre en question. Des fois, c'est important de se remettre en question, mais aussi de garder vraiment son point de vue, d'être sûre de savoir ce qu'on veut. De pas vouloir se conformer à quelque chose m'a aidée à me dire « je ne suis pas obligée de choisir tel programme si je sais que j'en préfère vraiment un autre ». [...] On vit dans un pays où nos droits sont respectés, puis on ne t'impose pas nécessairement des choix ou des trucs à faire. Ça, c'est une ressource qui pourrait m'aider là.

Les choix d'orientation se clarifient progressivement pour les membres. Zahra soutient qu'elle aimerait faire des sciences au Cégep et envisage une profession (qui demeure à préciser) dans le domaine de la santé. Pauline envisage de faire deux diplômes d'études collégiales, l'un en danse, sa grande passion, et l'autre en sciences, un domaine qu'elle aime également. Elle prévoit des stratégies pour en parler à ses parents, pour qu'ils approuvent éventuellement ce choix. Parisa nomme qu'elle se donne le droit de revenir à son rêve d'enfance d'être médecin de famille. Elle précise qu'elle veut ajouter du bénévolat pour aider d'autres personnes et elle reconnaît, comme cela lui a été reflété dans le groupe, sa capacité à le faire.

\section{Discussion et Conclusion}

La mise en œuvre et l'évaluation du programme S'Orienter ont permis de dégager des représentations sexospécifiques associées à des métiers et des professions et leur inscription dans des rapports de pouvoir asymétriques qui continuent d'affecter les possibilités professionnelles envisagées par des jeunes. Avec le programme, des instruments conceptuels ont été transmis pour soutenir l'orientation professionnelle, mais également pour faciliter la conscience des représentations sexospécifiques pouvant affecter les choix et projets scolaires et professionnels des personnes participantes. Dans une société où la norme d'égalité des sexes fait l'objet d'un consensus social (Marchand, Saint-Charles, \& Corbeil, 2007), nos résultats montrent sur ces deux sites que les représentations sexospécifiques sont difficiles à exprimer ou demeurent non-conscientes chez les jeunes, c'est-à-dire - dans la perspective de Vygotski (2013/1934) - qu'elles n'ont pas fait l'objet d'un travail de pensée verbale pour 
pouvoir être utilisées de manière consciente et volontaire. Au départ, les personnes participantes conçoivent que femmes et hommes disposent de la même liberté de choisir et réaliser leurs ambitions, ou elles sont invitées à se taire si elles affirment le contraire. Les réflexions suscitées dans le cadre du programme S'Orienter et les instruments langagiers transmis amènent les personnes participantes à mettre en perspective cette conception concernant l'égalité des sexes en matière de choix d'orientation scolaire et professionnelle. Les dimensions descriptives et prescriptives des représentations sexospécifiques décrites par Heilman (2012) sont mises en débat. Sur le site 1 , les discussions mènent les membres du groupe à concevoir que dans leur entourage, ou dans un environnement proximal, une infériorisation des capacités des femmes persiste, notamment eu égard à certains métiers liés à l'agriculture ou à la forêt. Sur le site 2, les jeunes femmes expriment une contradiction vécue entre la liberté réelle de mettre en œuvre leurs aspirations professionnelles et des pressions familiales les poussant à choisir des professions ayant un statut social élevé. Cette pression, vécue comme une contrainte à une liberté réelle d'orientation scolaire et professionnelle, spécifique au fait d'être une femme, rejoint d'autres travaux réalisés sur ce thème (Lalonde \& Giguère, 2008) auprès de jeunes femmes issues de l'immigration. Nos résultats permettent aussi de soutenir que le programme favorise la clarification, pour la majorité des personnes participantes, de leurs choix d'orientation scolaire et professionnelle.

26 L'une des limites du programme S'Orienter relève par ailleurs de sa durée (10 h), qui demeure relativement courte selon notre perspective. Pour soutenir toutes les étapes de réalisation d'un choix d'orientation et la transformation durable des représentations sexospécifiques, il serait souhaitable d'introduire des suivis au cours de l'année qui suit la participation au programme. Cela favoriserait sans doute une plus grande pérennité des retombées du programme. En outre, le modèle d'accompagnement mobilisé dans le programme, qui nécessite de la personne conseillère une capacité à partager le pouvoir et les décisions avec les membres, peut être déstabilisant pour elle ou pour les membres, comme cela a été vécu sur le site 2 . Ce mode de fonctionnement nécessite d'être abordé directement dans le cadre du programme pour éviter les ruptures d'alliance de travail avec les membres.

Enfin, comme le soutient Leclerc (2019), l'intervention de groupe offre un espace de soutien de l'expression et l'affirmation de soi qui peut favoriser l'exposition à des formes de pensées différentes en lien avec l'orientation. Les résultats que nous avons rapportés tendent à montrer que cela soutient progressivement un rapport plus conscient à soi, aux autres et au monde, particulièrement en lien avec les représentations sexospécifiques qui pouvaient affecter les choix d'orientation. Pour plusieurs membres, les retours fréquents sur leurs compétences et ressources ont favorisé un regard plus positif sur leur confiance dans leurs capacités à réaliser un choix porteur de sens pour eux, et ce, sans se limiter par le fait d'être une femme ou un homme. Nos résultats invitent enfin à réfléchir plus largement au rôle des interventions en orientation dans la perspective de contribuer à une société plus juste socialement et équitable en termes d'orientation scolaire et professionnelle. En plus des stéréotypes limitants au regard du sexe, nos données laissent entrevoir l'importance de les considérer en lien avec d'autres représentations qui peuvent entraver les possibilités de certains jeunes à s'orienter vers un projet professionnel porteur de sens 
pour eux, des représentations de l'appartenance culturelle, de l'origine sociale ou des capacités scolaires par exemple.

\section{BIBLIOGRAPHIE}

Aguiar, F. H. R., \& Conceição, M. I. G. (2015). Career guidance and health promotion with adolescents: keys for intervention programs. International Journal for Educational and Vocational Guidance, 15, 221-236. https://doi.org/10.1007/ s10775-015-9311-9

Ali, S. R., Brown, S. D., \& Loh, Y. (2017). Project HOPE: Evaluation of health science career education programming for rural latino and european american youth. The Career Development Quarterly, 65, 57-71. https://doi. org/10.1177\%2F0894845319832973

Bandura, A. (1986). Social foundations of thought and action: a social cognitive theory. New York, NJ: Prentice-Hall.

Bimrose, J., McMahon, M., \& Watson, M. (2019). Women and Social Justice: Does Career Guidance Have a Role? In T. Hooley, R. G. Sultana, \& R. Thomsen (Eds.), Career Guidance for Emancipation: Reclaimonig Justice for the Multitude (pp. 17-32). New York, NY: Routledge.

Chronister, K. M., McWhirter, E. W., \& Forrest, L. (2005). A critical feminist approach to career counseling with women. In B. W. Walsh \& M. J. Heppner (Eds.), Handbook of Career Counseling for Women (pp. 167-192). Mahway, NJ: Lawrence Erlbaum associates.

Dionne, P., Saussez, F., \& Bourdon, S. (2017) Reconversion et développement du pouvoir d'agir par l'apprentissage de systèmes d'action en groupe de réinsertion sociale et professionnelle. L'Orientation scolaire et professionnelle, 46(3). Repéré à https://doi.org/10.4000/osp.5475

Dionne, P., Simard, A., \& Bourdon, S., Supeno, E., \& Girardin, V. (2020). S'Orienter. Sherbrooke, Canada : Centre d'études et de recherches sur les transitions et l'apprentissage. Repéré à http:// www.erta.ca/index.php/fr/node/1062

Duru-Bellat, M. (2011). La face subjective des inégalités. Une convergence entre psychologie sociale et sociologie. Sociologie, 2, 185-200. https://doi.org/10.3917/socio.022.0185

Heilman, M. (2012). Gender stereotypes and workplace bias. Research in Organisational Behavior, 32, 113135. https://doi.org/10.1016/j.riob.2012.11.003

Hernandez, L., Oubrayrie-Roussel, N., \& Prêteur, Y. (2014). De l'affirmation de soi dans un groupe de pairs à la démobilisation scolaire. Enfance, 2(2), 135-157. https://doi.org/10.4074/ S001375451400202x

Kergoat, P. (2014). Le travail, l'école et la production des normes de genre. Filles et garçons en apprentissage (en France). Nouvelles questions féministes, 33(1), 16-34. https://doi.org/10.3917/nqf. 331.0016

Lalonde, R. N., \& Giguère, B. (2008). L'entrechoquement des univers culturels de la deuxième génération. Diversité Canadienne, 6(2), 65-69.

Leclerc, C. (2019). Intervenir en groupe : savoir et pouvoir d'agir. Québec, Canada : CRIEVaT. 
Marchand, I., Saint-Charles, J., \& Corbeil, C. (2007). L'ascension professionnelle et le plafond de verre dans les entreprises privées au Québec. Recherches féministes, 20(1), 27-54. https://doi.org/ 10.7202/016115ar

Metz, A. J., Fouad, N., \& Ihle-Helledy, K. (2009). Career aspirations and expectations of college students. Demographic and labor market comparisons. Journal of career assessment, 17(2), 155-171. https://doi.org/10.1177/1069072708328862

Nota, L., Soresi, S., \& Ferrari, L. (2008). « Premier principe : je crois en moi... parce que c'est aussi dans mon intérêt » : une formation pour renforcer les sentiments d'efficacité. L'Orientation scolaire et professionnelle, 37(1), 113134. https://doi.org/10.4000/osp.1628

Scott, A. B., \& Ciani, K. D. (2008). Effects of an undergraduate career class on men's and women's career decision-making self-efficacy and vocational identity. Journal of Career Development, 34(3), 263-285. https://doi.org/10.1177\%2F0894845307311248

Shea, M., Ma, P.-W. W., Yeh, C. J., Lee, S. J., \& Pituc, S. T. (2009). Exploratory studies on the effects of a career exploration group for urban chinese immigrant youth. Journal of career assessment, 17(4), 457-477. https://doi.org/10.1177\%2F1069072709334246

Statistique Canada. (2017). Les femmes et le travail rémunéré. Ottawa, Canada : Gouvernement du Canada.

Vouillot, F., Blanchard, S., Marro, C., \& Steinbrukner, M.-L. (2004). La division sexuée de l'orientation et du travail : une question théorique et une question de pratiques. Psychologie $\mathrm{du}$ travail et des organisations, 10, 277-291. https://doi.org/10.1016/j.pto.2004.07.004

Vouillot, F. (2007). Formation et orientation : l'empreinte du genre. Travail, genre et sociétés. 2(18), 23-26. https://doi.org/10.3917/tgs.018.0023

Vygotski, L. S. (2013/1934). Pensée \& langage (4éd.). Paris, France : La Dispute.

\section{NOTES}

1. Le vocable "représentation sexospécifique » est mobilisé ici sur la base de la binarité liée au sexe biologique des personnes. Ce regard sur le sexe est posé en amont de la définition ou du rapport d'identification que les personnes ont vis-à-vis de leur sexe biologique sur le plan identitaire. La sexospécificité s'inscrit dans une perspective de rapports sociaux entre les sexes. Ainsi, le vocable représentations sexospécifiques peut inclure le concept de genre pensé en tant que «système de normes de féminité/masculinité qui définit et prescrit ce que doivent être et faire les femmes et les hommes dans une société donnée » (Vouillot, 2007, p. 25). Par ailleurs pour cet article, nous ne disposons pas de données sur des personnes qui déclarent leur sexe comme non-binaire.

2. Pour plus de détails sur les activités spécifiques du programme et leur séquence, consulter (Dionne et al., 2020)

3. Chronister, McWhirter, \& Forrest (2005)

4. Chronister, McWhirter, \& Forrest (2005)

5. Ces dernières se sont vu attribuer un nom fictif pour garantir leur anonymat.

6. L'affirmation est vue au sens d'Hernandez, Ourbrayrie-Roussel et Prêteur (2014) comme une capacité à verbaliser son point de vue, même s'il diverge de celui d'un groupe, cela peut se traduire " par des prises d'initiatives, des capacités d'écoute et des capacités à répondre aux pressions des pairs, à exprimer ses idées, opinions et sentiments » (p. 138). 


\section{RÉSUMÉS}

Cet article porte sur l'implantation et les retombées du programme de groupe S'Orienter, visant à transformer les représentations sexospécifiques d'élèves du secondaire sur les métiers et les professions, représentations qui, en limitant les aspirations professionnelles, peuvent influencer l'orientation scolaire et professionnelle. Les analyses, menées dans une perspective culturelle historique de l'activité, ont permis de dégager que la participation au programme S'Orienter notamment par les débats introduits autour de représentations sexospécifiques - soutient chez les élèves le développement d'un rapport plus conscient à ces représentations et à la façon dont elles peuvent affecter leurs choix éducatifs et professionnels.

This article focuses on impacts of a career counseling group program, S'Orienter, aimed at generating students learning and empowerment on certain gender stereotypes, which, by limiting career aspirations, can influence academic and professional choices. The analyzes, carried out within a cultural-historical activity theoretical perspective, show how participation in the S'Orienter program, and the occasions to question gender stereotypes, gradually increase students' consciousness and empowerment on what restricts their academic and professional choice.

\section{INDEX}

Keywords : gender stereotypes, group career counseling, cultural-historical activity theory

Mots-clés : représentations sexospécifiques, orientation scolaire et professionnelle, programme de groupe, théorie culturelle-historique de l'activité

\section{AUTEURS}

\section{PATRICIA DIONNE}

Professeure agrégée, conseillère d'orientation, Département d'orientation professionnelle Université de Sherbrooke

Thèmes de recherche : analyse de l'activité, counseling de carrière groupal, orientation professionnelle d'adultes en situation de pauvreté et de précarité, immigration, bilan de compétences. Patricia.Dionne@usherbrooke.ca

\section{AMÉLIE SIMARD}

Doctorante en éducation (Ph. D), Conseillère d'orientation, Faculté d'éducation, Université de Sherbrooke

Thèmes de recherche : théorie culturelle-historique, analyse des processus langagiers, dynamique de groupe, counseling, orientation éducative et professionnelle. amelie.Simard@usherbrooke.ca

\section{SYLVAIN BOURDON}

Professeur titulaire, Département d'orientation professionnelle, Université de Sherbrooke Thèmes de recherche : transitions des jeunes adultes et des adultes peu scolarisés, trajectoires 
scolaires et persévérance aux études, formes alternatives de scolarisation, apprentissage tout au long de la vie. Sylvain.Bourdon@usherbrooke.ca 